\title{
Highly ordered good crystalline ZnO-doped WO3 thin films suitable for optoelectronic applications.
}

KAVITHA, V.S., BOSE, R.J., SREEDHARAN, R.S., SUDARSANAKUMAR, C., PRABHU, R. and PILLAI, V.P.M. 


\title{
Highly ordered Good crystalline $\mathrm{ZnO}$ doped $\mathrm{WO}_{3}$ thin films suitable for Optoelectronic applications
}

\author{
V.S. Kavitha ${ }^{\mathrm{a}}$, R. Jolly Bose ${ }^{\mathrm{a}}$, R. Sreeja Sreedharan ${ }^{\mathrm{a}}$, C. Sudarsanakumar ${ }^{\mathrm{b}}$, Radhakrishna \\ Prabhuc, V.P. Mahadevan Pillai ${ }^{\mathrm{a}^{*}}$ \\ ${ }^{a}$ Department of Optoelectronics, University of Kerala, Kariavattom, Thiruvananthapuram- \\ 695581, Kerala, India. \\ ${ }^{b}$ School of Pure and Applied Physics, Mahatma Gandhi University, Priyadarsini Hills, \\ Kottayam 686560, Kerala, India. \\ 'School of Engineering, Robert Gordon University, Aberdeen, UK. \\ *Email-vpmpillai9@gmail.com
}

\begin{abstract}
Highly ordered $\mathrm{ZnO}$ doped $\mathrm{WO}_{3}$ thin films with good crystalline quality are prepared using RF magnetron sputtering technique and its structural and morphological properties are studied using various characterisation tools such as XRD, micro-Raman, XPS, FESEM and EDX analysis. X-ray diffraction studies reveal the enhancement of crystalline quality of the films with increase in $\mathrm{ZnO}$ doping concentration up to 5 wt\%, beyond which the crystalline quality gets deteriorated. A phase modification from single monoclinic $\mathrm{WO}_{3}$ phase to a mixed monoclinic $\mathrm{WO}_{3}$ and $\mathrm{W}_{18} \mathrm{O}_{49}$ phases is observed for films with higher $\mathrm{ZnO}$ doping concentrations. Morphological analysis shows that a smooth surface for pure film whereas the ZnO doped films presents a dense distribution of grains of larger sizes with well-defined grain boundary.
\end{abstract}

\section{Introduction}

Tungsten trioxide $\left(\mathrm{WO}_{3}\right)$ is an important semiconductor oxide material showing a broad spectrum of novel properties [1]. It shows structural and stoichiometric transitions upon change in conditions that fascinated scientists to explore their future technological applications in different fields [2-3]. Since structural and morphological properties of nanomaterials show a vital role in device performance, the designing of nanomaterials is a crucial problem when technological applications are concerned [4]. From application point of view, it is utmost desirable to synthesise highly crystallized nanomaterial thin films by controlling their structural and morphological properties. $\mathrm{WO}_{3}$ thin films are better known for their important physiochemical properties which make them a suitable candidate in catalysis, gas sensors, electro-chromic devices, etc. [5-7]. The structural and morphological properties of $\mathrm{WO}_{3}$ thin films (at sub-micrometric scale) have profound effects on its physiochemical properties, which strongly depends on its preparation methods and conditions [8]. $\mathrm{WO}_{3}$ thin films have been prepared by many groups through different techniques like chemical vapour deposition[9], RF magnetron sputtering [10], solvothermal process [11], electrodeposition [12], sol-gel process [13], hydrothermal techniques [14], atomic layer deposition [15], pulsed laser deposition technique [16-17], etc. Among this RF magnetron sputtering technique is relatively simple and one can grow homogenous, high quality thin films of larger area suitable for device applications. Here we report the fabrication of $\mathrm{ZnO}$ doped $\mathrm{WO}_{3}$ thin 
films of superior crystalline quality using RF magnetron sputtering technique and its structural and morphological studies using different techniques such as XRD, micro-Raman, XPS, FESEM and EDX analysis.

\section{Experimental details}

ZnO doped tungsten oxide thin films were prepared using RF magnetron sputtering technique from sputtering targets made of $\mathrm{WO}_{3}$ powder (Sigma, purity 99.99\%) and desired doping concentrations (0, 0.5, 1, 3, 5 and 7 wt\%) of ZnO powder (Sigma, purity 99.99\%). Chamber pressure was maintained initially at $10^{-6}$ mbar using a diffusion pump and a rotary pump and then pure argon gas (argon pressure of 0.015 mbar) was introduced in to the chamber during deposition. Films were fabricated on quartz plate (kept at $5 \mathrm{~cm}$ from the target) with a constant RF power of 150 watts for a deposition time of 30 minutes. The as-deposited films are annealed at a temperature $600^{\circ} \mathrm{C}$ for 1 hour. These films with ZnO doping concentrations 0, 0.5, 1, 3, 5 and 7 wt\% are coded as WZ0, WZ0.5, WZ1, WZ3, WZ5 and WZ7 respectively. XPERT PRO Diffractometer (Bruker) was employed to perform the structural analysis of the films using Bragg-Brentano geometry. CuK $\alpha 1 \mathrm{X}$-ray radiation of wavelength $1.5406 \AA$ was used and the XRD patterns were recorded for the $2 \theta$ range of $20-70^{\circ}$ with a step size of 0.020305 and at a scan speed of $2^{\circ} \mathrm{min}^{-1}$. Labram-HR800 spectrometer (Horiba Jobin Yvon, USA) equipped with an Argon-ion laser (wavelength $514.5 \mathrm{~nm}$ ) was used to record the Raman spectra (spectral resolution $\sim 1$ $\mathrm{cm}^{-1}$ ). The valance state and stoichiometry of the deposited films are studied using X-ray photoelectron spectrometer equipped with a $A 1 K_{\alpha}$ monochromatic X-ray source of $\sim 1486.7 \mathrm{eV}$ energy (Omicron Technology). The surface analysis and elemental analysis of the films were done using a Nova Nano SEM-450 Field Emission Scanning Electron Microscope (FEI- USA) equipped with an XFlash detector 6/10 (Bruker) and electron energy dispersive X-ray spectrometer (EDS-Quantax 200, Germany). The average thickness of the films was determined using lateral FESEM images.

\section{Results and discussions}

\subsection{XRD Analysis}



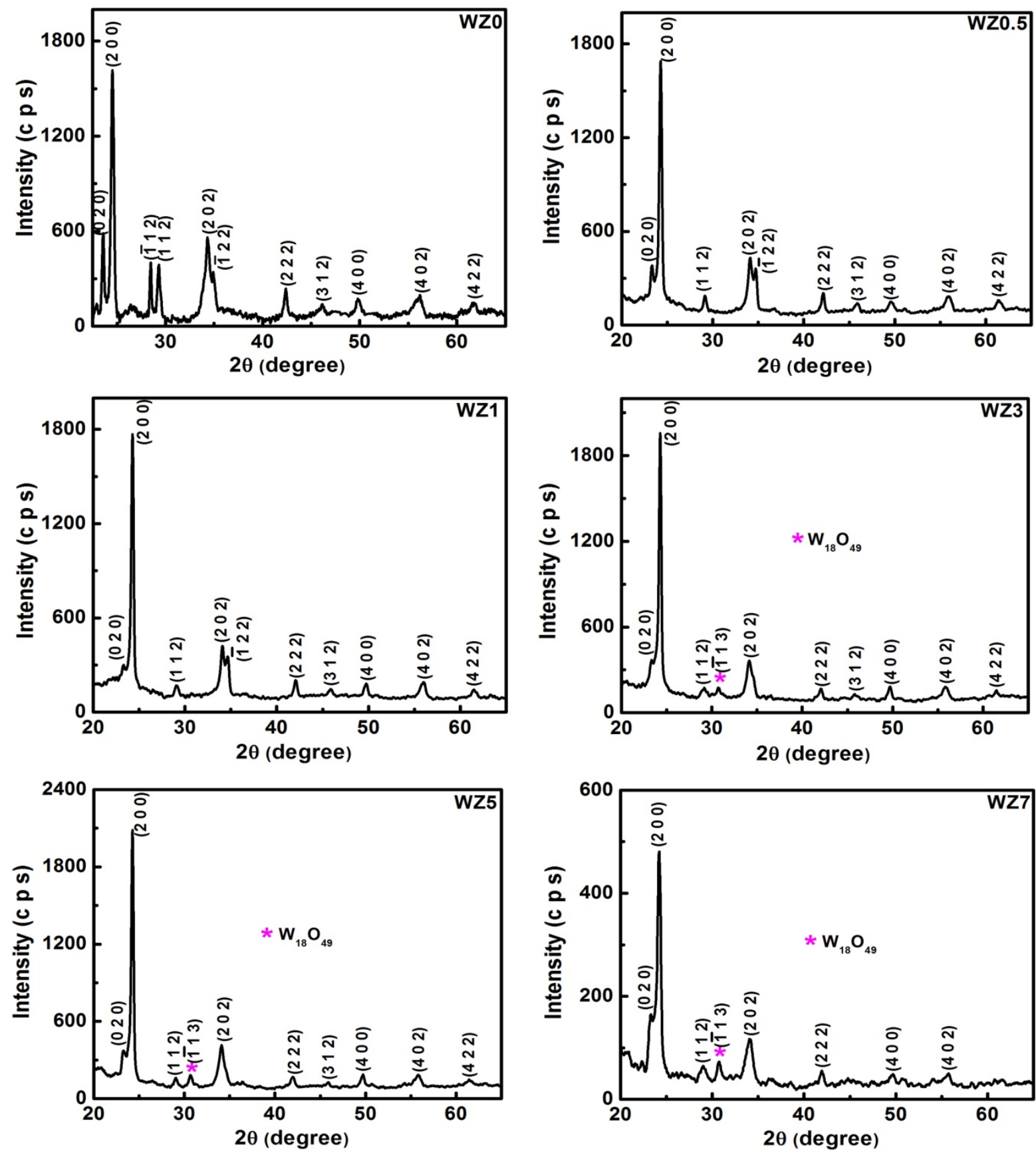

Fig.1. XRD patterns of the pure and $\mathrm{ZnO}$ doped $\mathrm{WO}_{3}$ thin films annealed at a temperature of $600{ }^{\circ} \mathrm{C}$

X-Ray diffraction patterns of the pure and $\mathrm{ZnO}$ doped tungsten oxide thin films annealed at a temperature of $600^{\circ} \mathrm{C}$ are presented in Fig.1. The XRD patterns reveal that all the post-annealed films are polycrystalline in nature. Films with 0, 0.5 and $1 \mathrm{wt} \% \mathrm{ZnO}$ doping present the characteristic diffraction peaks of only monoclinic $\mathrm{WO}_{3}$ crystalline phase (JCPDS Card No. 83-0951). Thus the pure and lightly ZnO doped films exhibit a single crystalline phase. However the XRD patterns of the higher $\mathrm{ZnO}$ doped $\mathrm{WO}_{3}$ films present mixed crystalline phases. For these films in addition to the peaks due to the monoclinic $\mathrm{WO}_{3}$ phase an additional peak $(\overline{1} 13)(2 \theta$ $=30.6^{\circ}$ ) corresponding to monoclinic $\mathrm{W}_{18} \mathrm{O}_{49}$ (JCPDS Card No. 84-1516) phase also observed whose intensity enhances with increase in $\mathrm{ZnO}$ doping concentration. This indicates that higher $\mathrm{ZnO}$ doping concentration 
favours a phase modification from single monoclinic $\mathrm{WO}_{3}$ phase to a mixed monoclinic $\mathrm{WO}_{3}$ and $\mathrm{W}_{18} \mathrm{O}_{49}$ phases. In our earlier studies we have observed silver incorporation in $\mathrm{WO}_{3}$ promotes the formation of crystalline $\mathrm{W}_{18} \mathrm{O}_{49}$ monoclinic phase [18]. Also we have observed a phase modification from triclinic $\mathrm{WO}_{3}$ phase to monoclinic $\mathrm{W}_{18} \mathrm{O}_{49}$ phase due to gold incorporation in $\mathrm{WO}_{3}$ lattice [10]. Even though the films with higher ZnO doping concentration present a mixed phase, the large intensity of the peaks along $<200>$ direction in the prepared films confirm the dominance of monoclinic $\mathrm{WO}_{3}$ crystalline phase in all the films. Thus it is found that all the films show an a-axis preferential growth which may be due to the least value of surface free energy of the crystal along a-axis that can lead to self-ordering effects [19]. This preferential growth along a-axis can be explained by the "survival of the fastest" model (by Van der Drift) which states that in the initial stage of the film growth nucleation with different orientation can be formed and these nuclei compete to grow. Finally the nucleus with higher growth rate can survive leading the film growth along a-axis. [20-21]. The $\mathrm{ZnO}$ doping in $\mathrm{WO}_{3}$ lattice seems to have considerable effect on the crystalline nature of the films. The intensity of the (200) peak shows a systematic increase with increase in $\mathrm{ZnO}$ doping concentration up to 5 $\mathrm{wt} \%$. Beyond $5 \mathrm{wt} \%$ the increase in $\mathrm{ZnO}$ doping concentration produces a drastic decrease in intensity of the (200) peak. The full width at half maximum (FWHM) of the (200) peak for the pure $\mathrm{WO}_{3}$ film is $0.3268^{\circ}$. Interestingly the value of the FWHM of (200) peak for the ZnO doped films shows a systematic decrease with increase in $\mathrm{ZnO}$ doping concentration up to $5 \mathrm{wt} \%$ and thereafter its FWHM increases with increase in $\mathrm{ZnO}$ doping concentration (Fig 2(a)). This observed increase in the intensity of (200) peak up to 5 wt\% may be due to the formation of new nucleating centres developed because of the added impurity. Following this, a decrease in the (200) peak intensity at higher doping concentration can be due to the saturation of newer seeding sites and the segregation of dopant impurity along the grain boundaries at higher doping concentrations [21-22]. These observations indicate that moderate doping of $\mathrm{ZnO}$ in $\mathrm{WO}_{3}$ lattice improves its crystalline quality. Thus it is clear that $5 \mathrm{wt} \% \mathrm{ZnO}$ doping is the optimum doping concentration for producing good quality crystalline $\mathrm{WO}_{3}$ films. It is interesting to note that the XRD pattern of pure film presents a medium intense peak along $<\overline{1} 12>$ direction but in all the ZnO doped films this peak remains absent. Also in the XRD pattern of WZ0 film (020) peak is observed with medium intensity. With $\mathrm{ZnO}$ doping in $\mathrm{WO}_{3}$ the intensity of this peak reduced considerably and it appears either as a weak peak or as a shoulder peak in the XRD patterns of all the ZnO doped films. The medium intense peaks (112), (202) and (222) observed in WZ0 film also show considerable reduction in intensity with $\mathrm{ZnO}$ doping. These observations tell us that $\mathrm{ZnO}$ doping in $\mathrm{WO}_{3}$ lattice promotes the preferential crystalline growth along $<200>$ direction and thus an a-axis orientation of the films.

FWHM ( $\beta_{h k l}$ ) of the most intense peak (200) in the XRD patterns is used to calculate the mean crystallites size ( $\left.D_{h k l}\right)$ of the films using the Scherrer formula [23]

$$
D_{h k l}=\frac{k \lambda}{\beta_{h k l} \cos \theta_{h k l}}
$$


where $k$ is a geometric constant called Scherrer constant and its value is usually taken as 0.9 for spherical crystallites and $\lambda$ is the wavelength of the $\mathrm{X}$-ray used. The mean crystallite size calculated for the films using the above equation are given in Table 1. The average size of the crystallites in the film increases with $\mathrm{ZnO}$ doping concentration up to $5 \mathrm{wt} \%$ and thereafter it decreases with higher $\mathrm{ZnO}$ doping concentrations. This reduction in crystallite size with higher $\mathrm{ZnO}$ content may be due to the hindering force generated due to the segregation of dopant impurity along the inter particles boundary and when this retarding force exceeds the driving force for the grain growth due to $\mathrm{W}$, the movement of the grain boundary is obstructed and thus decreasing the crystallite size [24-25].

In thin films residual stresses and corresponding strains always occur during the film synthesis. A measure of the lattice strain ie, lattice constant distribution originating from crystal imperfections and the measure of the crystallite size ie, the finite size of a coherently scattering domain affect the diffraction peaks and these effects may increase the integral width of the diffraction peak, change the diffraction peak intensity and also shift the centre of the diffraction peak [26]. Hence, the effects of the crystallite size induced broadening and the strain induced broadening in the FWHM of the diffraction peaks can be analysed by the following Williamson-Hall relation [27]

$\beta_{h k l} \cos \theta_{h k l}=\frac{k \lambda}{D_{h k l}^{\prime}}+2 \eta \sin \theta_{h k l}$

Williamson-Hall plots are drawn for the films (Fig.2(b)), the size of the strain free crystallites $D_{h k l}^{\prime}$ and the residual strain $\eta$ in the films are calculated using the plots and are tabulated in the Table 1 . The crystallite size estimated from the $\mathrm{W}-\mathrm{H}$ plots is higher than the values obtained from Scherrer equation. This suggests the presence of appreciable amount of lattice strain in the prepared films [28].
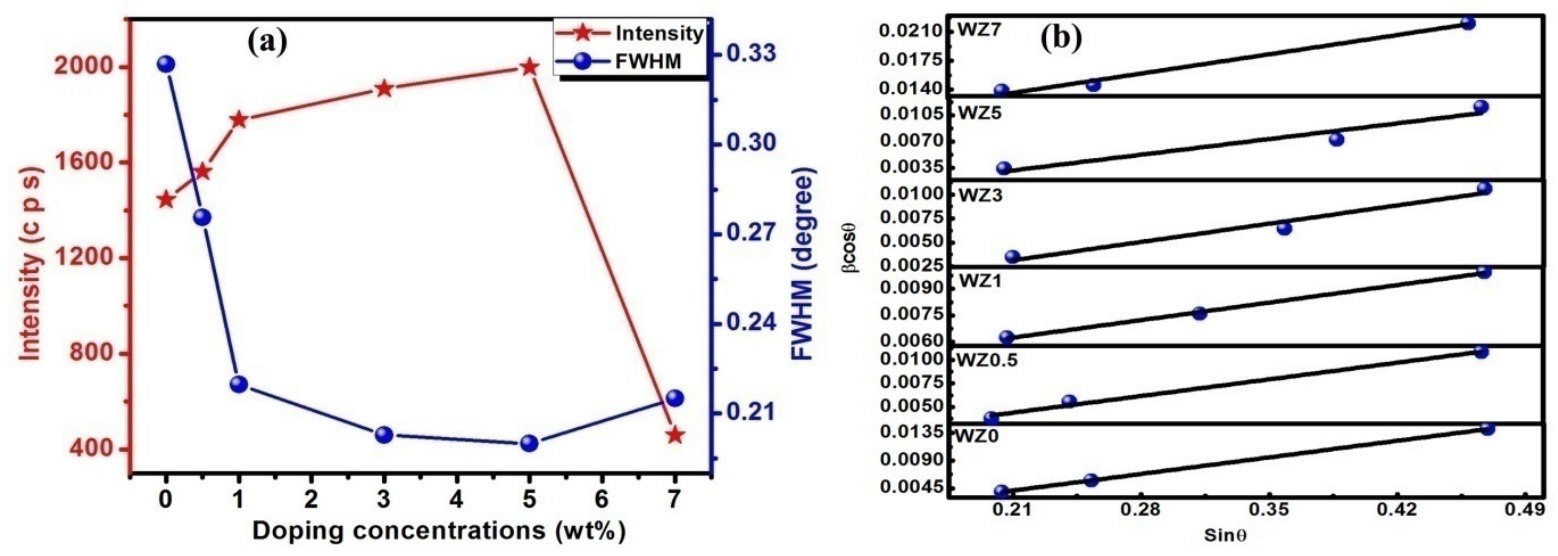

Fig.2. (a): Variations of intensity and FWHM of the most intense (200) peak with Zn doping concentration; (b): W-H plots of the pure and $\mathrm{Zn}$ doped $\mathrm{WO}_{3}$ thin films.

A zoomed view of the maximum intense peak (200) shows that the centre of the diffraction peak shifts towards lower diffraction angle with $\mathrm{ZnO}$ doping (Fig.3(a)). This shift to lower diffraction angle is reasonable to expect 
in the present case because the ionic radius of the $\mathrm{Zn}^{2+}(0.074 \mathrm{~nm})$ ion is slightly greater than $\mathrm{W}^{6+}(0.065 \mathrm{~nm})$ ion [29] which is an indication of the expansion of the unit cell due to the substitutional incorporation of $\mathrm{Zn}^{2+}$ ions at $\mathrm{W}^{6+}$ ionic sites (Vegard's law) [30-31]. The shift of the most intense peak to lower diffraction angle indicates the presence of residual stress in the films and this can be due to various reasons such as ionic size difference between $\mathrm{W}^{6+}$ and $\mathrm{Zn}^{2+}$, difference of thermal expansion coefficients (during annealing) and lattice constants between the film and the substrate, presence of defects and impurities etc. [32]. Lattice parameters (a, b and c) of the prepared films are calculated and are given in Table 1. As can be seen, the values of lattice parameters a, b and c show an increase with increase in $\mathrm{ZnO}$ doping concentration (Fig.3 (b) to (d) and Table 1). The interplanar spacing corresponding to (200) plane, $d_{200}$ is also calculated (Fig. 3(a) and Table 1) and it increases with increase in $\mathrm{ZnO}$ doping concentration. The increase in lattice parameters (a, b and c) and interplanar spacing $\left(d_{200}\right)$ with increase in $\mathrm{ZnO}$ doping concentration also supports the incorporation of $\mathrm{Zn}^{2+}$ ions in the $\mathrm{WO}_{3}$ host lattice and the resultant expansion of lattice [33].
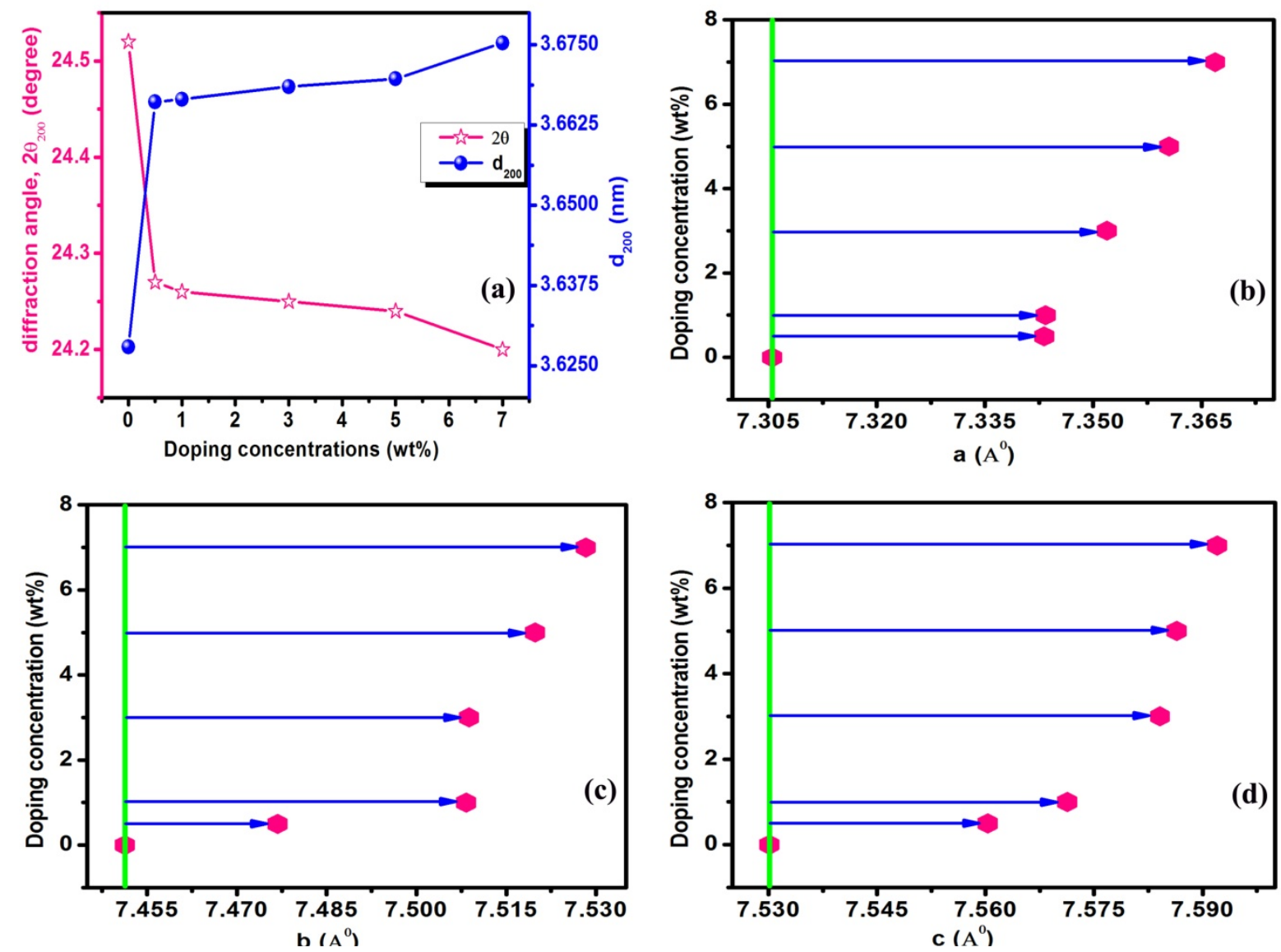

Fig.3.(a):Variations of diffraction angle $\left(2 \theta_{200}\right)$ and interplanar spacing $\left(d_{200}\right)$ of the most intense (200) peak as a function

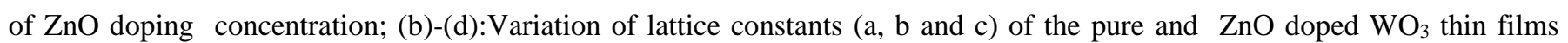
with doping concentrations. 
Table 1. Structural parameters of the pure and $\mathrm{ZnO}$ doped $\mathrm{WO}_{3}$ thin films annealed at a temperature of $600{ }^{\circ} \mathrm{C}$.

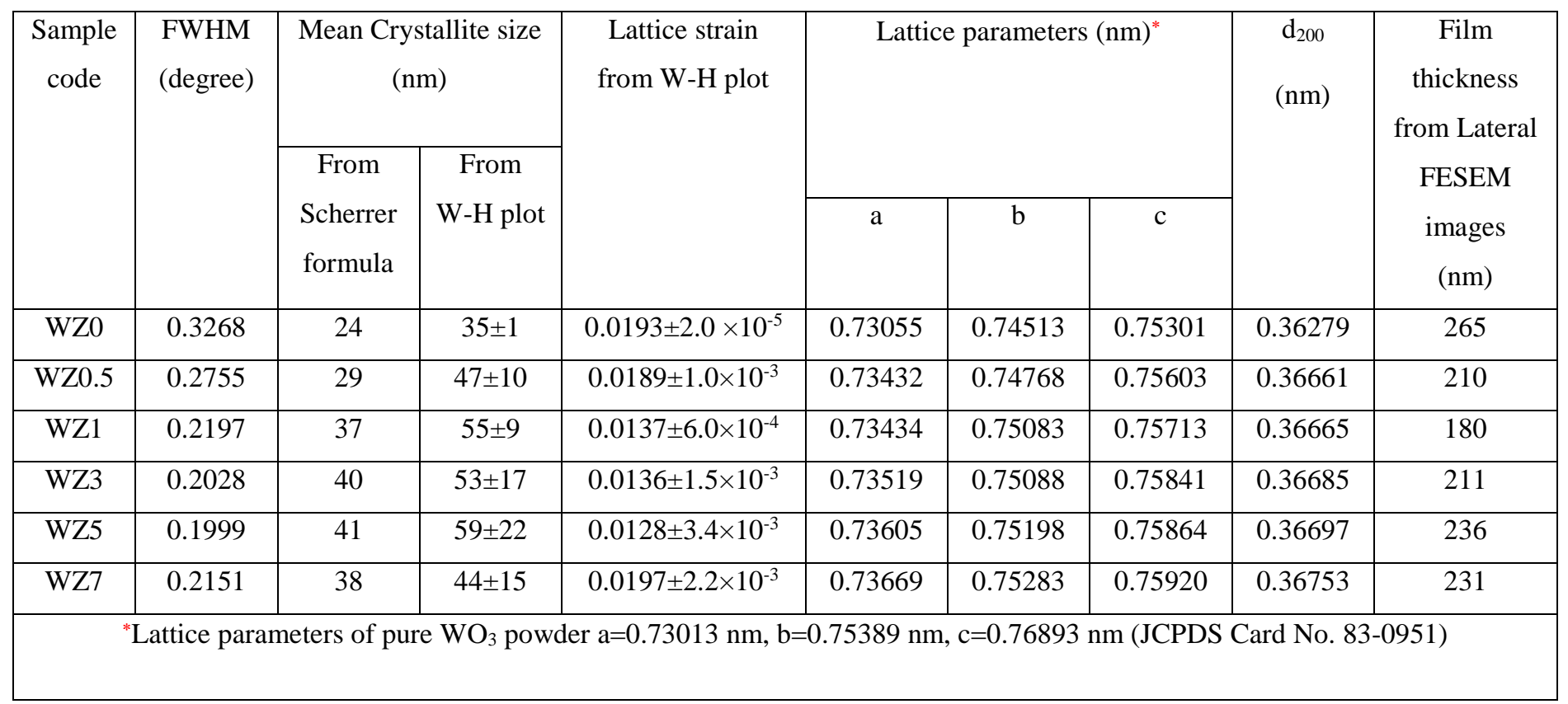

\subsection{Micro-Raman Analysis}



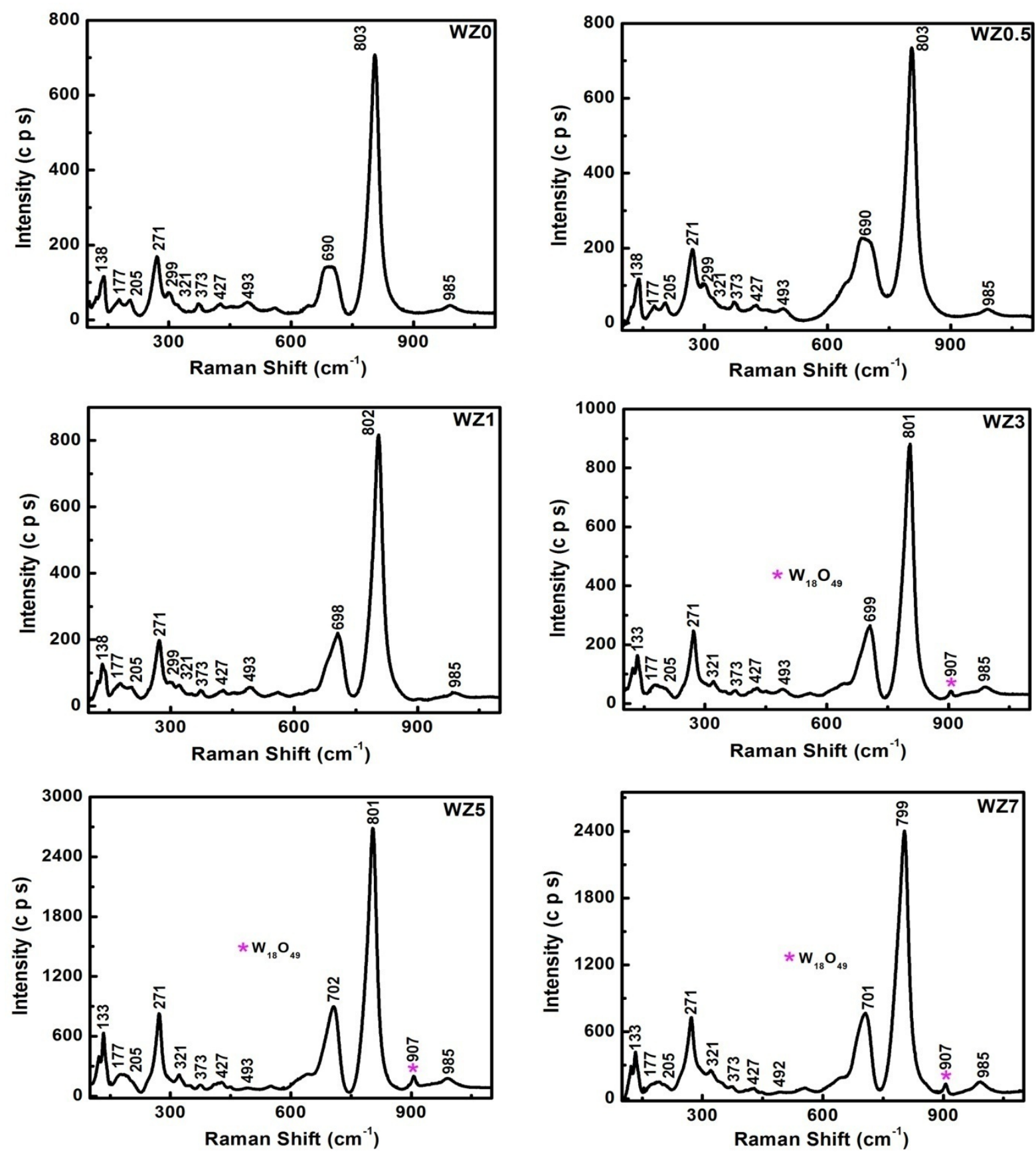

Fig.4. Micro-Raman spectra of the pure and $\mathrm{ZnO}$ doped $\mathrm{WO}_{3}$ thin films annealed at a temperature of $600{ }^{\circ} \mathrm{C}$.

The micro-Raman spectra of the pure and $\mathrm{ZnO}$ doped $\mathrm{WO}_{3}$ thin films (Fig.4) are recorded in the wavenumber region 100-1100 $\mathrm{cm}^{-1}$. In the Raman spectra of $\mathrm{WO}_{3}$ films vibrational bands below $200 \mathrm{~cm}^{-1}$ can be assigned to lattice modes, and the mid (200-500 $\left.\mathrm{cm}^{-1}\right)$ and high-wave number regions $\left(600-900 \mathrm{~cm}^{-1}\right)$ can be assigned to deformation and stretching modes of $\mathrm{WO}_{3}$ respectively [34-36]. For WZ0, WZ0.5 and WZ1 films the Raman spectra present a very intense band around $803 \mathrm{~cm}^{-1}$ and a medium intense band around $690 \mathrm{~cm}^{-1}$ being due to the symmetric and asymmetric stretching modes of $\mathrm{W}^{6+}-\mathrm{O}$ bond respectively. The bending vibrations of $\mathrm{O}-\mathrm{W}-\mathrm{O}$ bond are detected as several bands of less intensity in the 200-500 $\mathrm{cm}^{-1}$ region and an intense band around $271 \mathrm{~cm}^{-1}$. The presence of well-defined $\mathrm{WO}_{3}$ characteristic bands in the Raman spectra of these films 
indicates the formation of $\mathrm{WO}_{3}$ crystalline phase in these films. The Raman bands observed around 803, 702, and $272 \mathrm{~cm}^{-1}$ are characteristic vibrational modes of the crystalline $\mathrm{WO}_{3}$ monoclinic phase [2, 37-39]. So it is clear from figure that these films (WZ0, WZ0.5 and WZ1) have only crystalline monoclinic $\mathrm{WO}_{3}$ phase. The symmetric stretching mode shows a red shift from $803 \mathrm{~cm}^{-1}$ to $799 \mathrm{~cm}^{-1}$ with increase in ZnO doping concentration. The crystallite size calculation using Scherrer formula shows an increase of crystallite size with increase in $\mathrm{ZnO}$ doping concentration. Hence the observed shift in the symmetric stretching mode $\left(\gamma \mathrm{W}^{6+}-\mathrm{O}\right)$ is in accordance with the quantum confinement effect due to particle size variation. All the films present a weak band around $985 \mathrm{~cm}^{-1}$ region which can be attributed to the stretching of $\mathrm{W}^{6+}=\mathrm{O}$ bond. This unsaturated bond can be usually observed at the surfaces of clusters and voids in thin films $[7,40]$.

On increasing the $\mathrm{Zn}$ doping concentration from $1 \mathrm{wt} \%$ an additional band positioned around $907 \mathrm{~cm}^{-1}$ was observed in the Raman spectra whose intensity increases with increase in ZnO doping concentration. In our earlier investigations on $\mathrm{Ag}$ incorporated $\mathrm{WO}_{3}$ films and $\mathrm{Au}$ incorporated $\mathrm{WO}_{3}$ films a Raman band $\sim 917 \mathrm{~cm}^{-1}$ was observed for the stretching vibration of $\mathrm{W}_{18} \mathrm{O}_{49}$ phase $[10,18]$. The position of this band can be shifted to lower wavenumbers when the strength of the W-O bond decreases and the observed Raman band around 907 $\mathrm{cm}^{-1}$ in the Raman spectra of higher $\mathrm{ZnO}$ doped $\mathrm{WO}_{3}$ films can be assigned to the symmetric stretching vibration of $\mathrm{W}_{18} \mathrm{O}_{49}$ crystalline phase. Thus the result from the Raman spectral analysis is also in agreement with the results of the XRD analysis. So it can be concluded from XRD and Raman analysis that the pure $\mathrm{WO}_{3}$ films and the $\mathrm{WO}_{3}$ films with low $\mathrm{ZnO}$ doping concentrations (ie; 0.5 and $1 \mathrm{wt} \%$ ) contain only single monoclinic $\mathrm{WO}_{3}$ phase whereas in films with high $\mathrm{ZnO}$ doping concentrations (ie; 3, 5 and 7 wt\%) both monoclinic $\mathrm{WO}_{3}$ and monoclinic $\mathrm{W}_{18} \mathrm{O}_{49}$ phases coexist. However, in the films with higher $\mathrm{ZnO}$ doping concentration also, the monoclinic $\mathrm{WO}_{3}$ phase remains as the dominant crystalline phase.

\subsection{XPS Analysis}



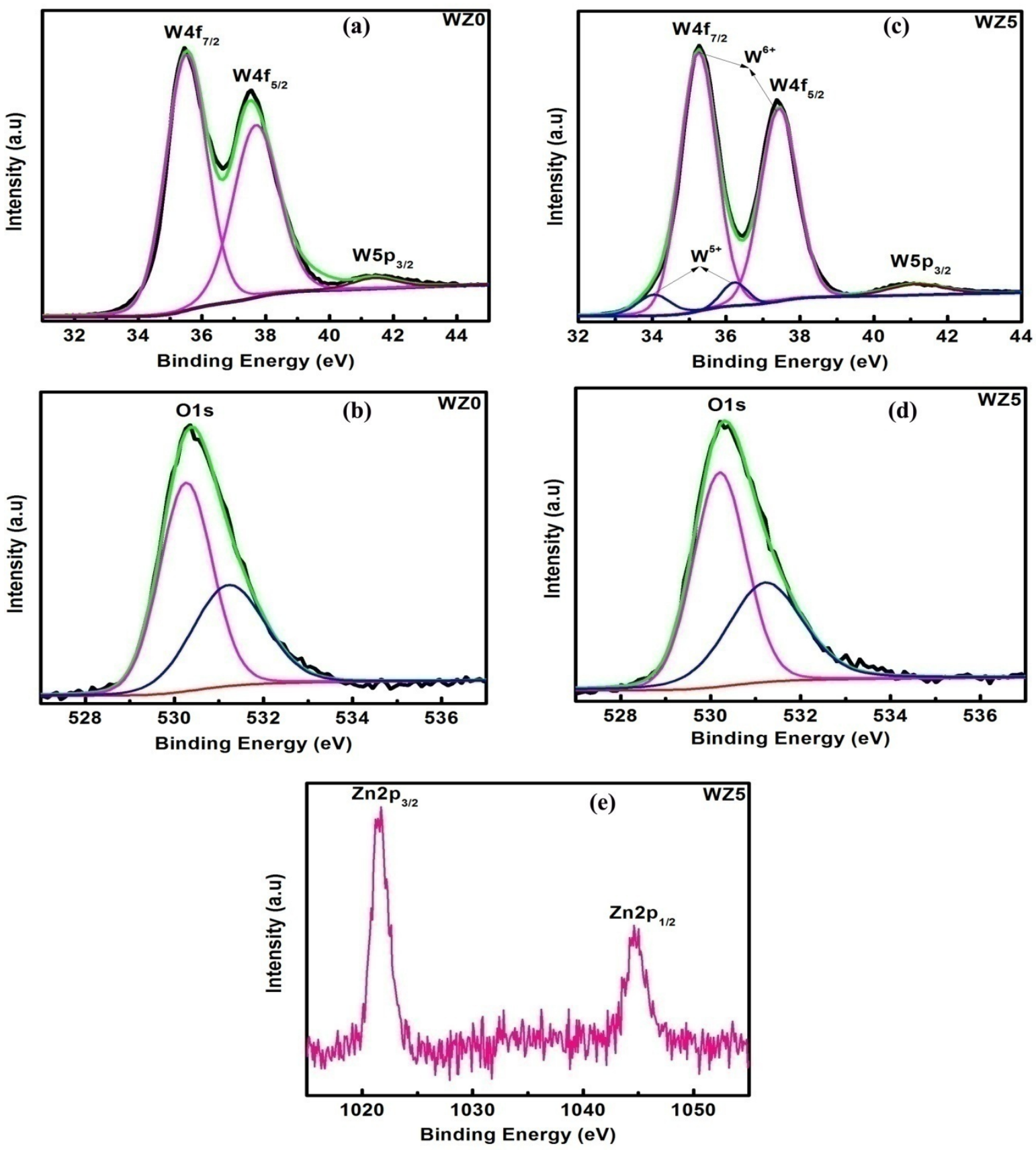

Fig.5.(a) and (c):W4f and $\mathrm{W}^{5} \mathrm{p}_{3 / 2}$ core level spectra of the pure and $5 \mathrm{wt} \% \mathrm{ZnO}$ doped $\mathrm{WO}_{3}$ thin films respectively; (b) and (d):O1s core level spectra of the pure and $5 \mathrm{wt} \% \mathrm{ZnO}$ doped $\mathrm{WO}_{3}$ thin films respectively; (e):Zn2p core level spectrum of the $5 \mathrm{wt} \% \mathrm{ZnO}$ doped $\mathrm{WO}_{3}$ thin film.

To study the surface stoichiometry, valance state and binding energy of the constituent elements in the deposited films, X-ray photoelectron spectroscopic measurement was carried out. XPS survey spectrum for the pure (WZ0) film (figure not included) shows the presence of $\mathrm{W}, \mathrm{O}$ and $\mathrm{C}$ elements but the film with $5 \mathrm{wt} \% \mathrm{ZnO}$ concentration (WZ5) shows the presence of Zn element as well (figure not included). The C1s peaks detected in the survey spectra may have the origin from the adventitious carbon containing molecules present on the sample surface that may have happened when the sample was exposed to air [41]. High resolution W4f and W5p $3 / 2$ core 
level spectra for the pure and $5 \mathrm{wt} \% \mathrm{ZnO}$ doped $\mathrm{WO}_{3}$ thin films are presented in Fig. 5(a) and (c) respectively. For pure film the W4f component can be fitted with a doublet, peaking around $35.52 \mathrm{eV}\left(\mathrm{W} 4 \mathrm{f}_{7 / 2}\right)$ and $37.68 \mathrm{eV}$ (W4f $\mathrm{f}_{5 / 2}$ ) while the $\mathrm{W}_{5} \mathrm{p}_{3 / 2}$ component can be fitted with a singlet, peaking around $41.42 \mathrm{eV}$ [42]. The observed binding energies of the doublet peaks $\mathrm{W}_{4} \mathrm{f}_{7 / 2}$ and $\mathrm{W}_{4} \mathrm{f}_{5 / 2}$ in undoped film is consistent with +6 valance state of $\mathrm{W}$ atom in $\mathrm{WO}_{3}$ [42]. The asymmetric O1s core level spectrum of the pure film presented in Fig. 5(b) can be deconvoluted in to two symmetric Gaussian peaks with binding energies $530.25 \mathrm{eV}$ and $531.22 \mathrm{eV}$. The highest intensity peak at $530.25 \mathrm{eV}$ suggests the presence of $\mathrm{O}^{2-}$ ion which is bonded to $\mathrm{W}^{6+}$ ion. The shoulder peak at $531.22 \mathrm{eV}$ arises due to the presence of oxygen adsorbed on the film surface [43]. The W4f and W5 $\mathrm{p}_{3 / 2}$ high resolution core level spectrum for the $5 \mathrm{wt} \% \mathrm{ZnO}$ doped film is shown in Fig. 5(c). Here the W4f region can be fitted with two doublets and the $\mathrm{W}_{5} \mathrm{p}_{3 / 2}$ region can be fitted with a singlet. The intense doublet peaks observed at $35.25\left(\mathrm{~W}_{4} \mathrm{f}_{7 / 2}\right)$ and $37.43 \mathrm{eV}\left(\mathrm{W}_{4} \mathrm{f}_{5 / 2}\right)$ correspond to +6 oxidation state of $\mathrm{W}$ atom [42] while the weak doublet peaks observed at $34.05\left(\mathrm{~W}_{4} \mathrm{f}_{7 / 2}\right)$ and $36.23 \mathrm{eV}\left(\mathrm{W}_{4} \mathrm{f}_{5 / 2}\right)$ corresponds to +5 oxidation state of $\mathrm{W}$ atom [44]. Even though we have fitted the $\mathrm{W} 4 \mathrm{f}$ region of $5 \mathrm{wt} \% \mathrm{ZnO}$ doped $\mathrm{WO}_{3}$ films with the spin orbit doublets corresponding to +6 and +5 oxidation state of $\mathrm{W}$ atom we can't rule out the existence of small amount of +4 oxidation state of W atom. These results lead to the conclusion that WZ5 film contains a trace amount sub oxide phase of $\mathrm{WO}_{3}\left(\mathrm{ie} ; \mathrm{W}_{18} \mathrm{O}_{49}\right.$ ) [45] which is in consistent with our earlier XRD and micro-Raman analysis. Here also (ie; for WZ5 film) the O1s peak (Fig. 5 (d)) can be fitted to two binding states ; one at low binding energy $(530.20 \mathrm{eV})$ corresponds to oxygen in $\mathrm{WO}_{3}$ structure and the other at high binding energy $(531.21 \mathrm{eV})$ corresponds to the surface adsorbed oxygen in the film [43]. Fig .5(e) shows the high resolution XPS spectrum of $\mathrm{Zn2p}$ core level in $5 \mathrm{wt} \% \mathrm{ZnO}$ doped $\mathrm{WO}_{3}$ film which shows a doublets at binding energy values 1021.74 and $1044.65 \mathrm{eV}$. The binding energy peaks at 1021.74 and $1044.65 \mathrm{eV}$ can be respectively assigned to Zn2p $3 / 2$ and $\mathrm{Zn} 2 \mathrm{p}_{1 / 2}$ energy levels in fully oxidized state of $\mathrm{Zn}$ atom ie; +2 oxidation state $\left(\mathrm{Zn}^{2+}\right)$ [46-48]. So it can be concluded that in our case the Zn dopant exist mainly in +2 oxidation state.

\subsection{FESEM and EDX Analysis}



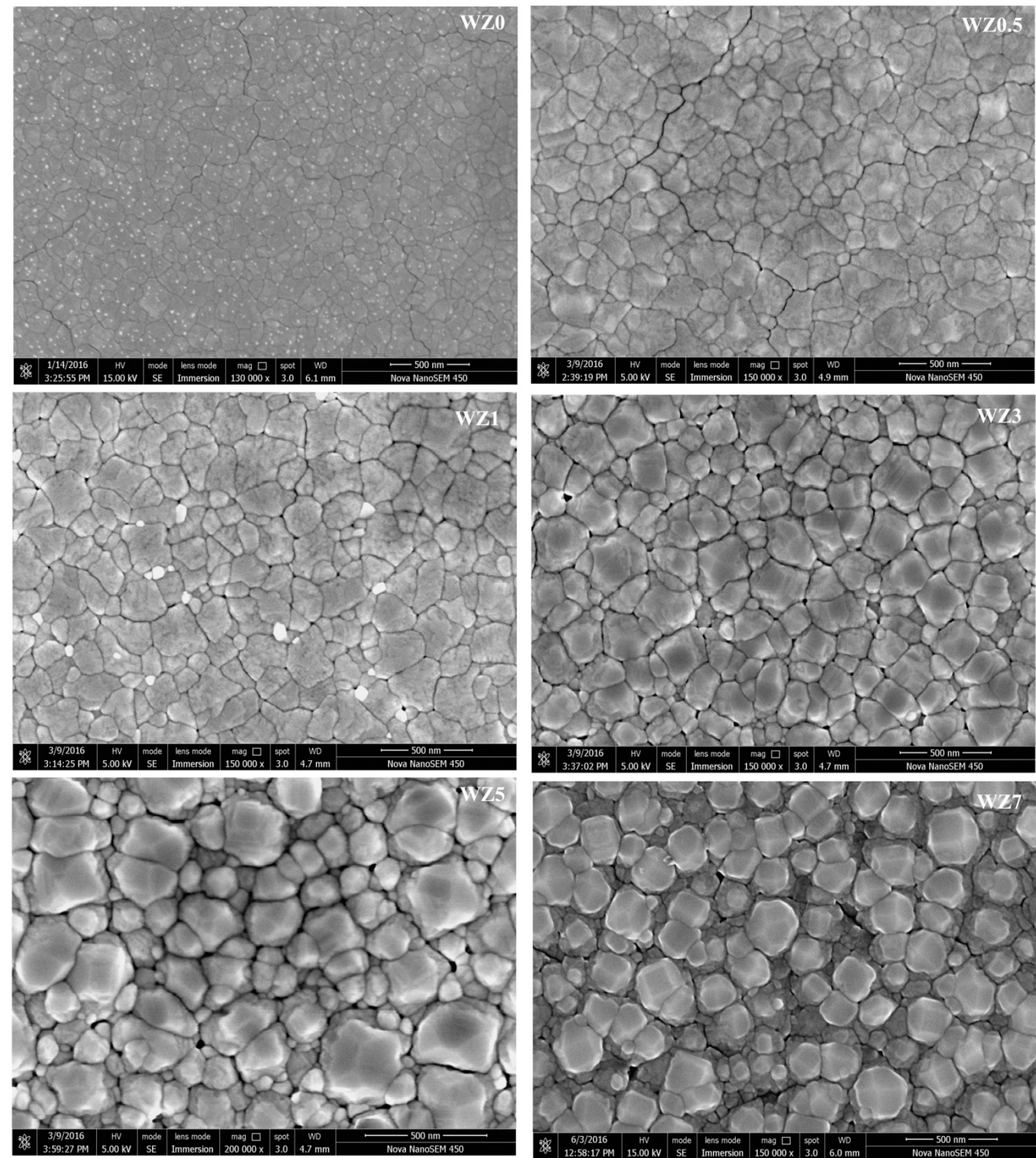

Fig. 6. FESEM micrographs of the pure and $\mathrm{ZnO}$ doped $\mathrm{WO}_{3}$ thin films annealed at a temperature of $600{ }^{\circ} \mathrm{C}$. 

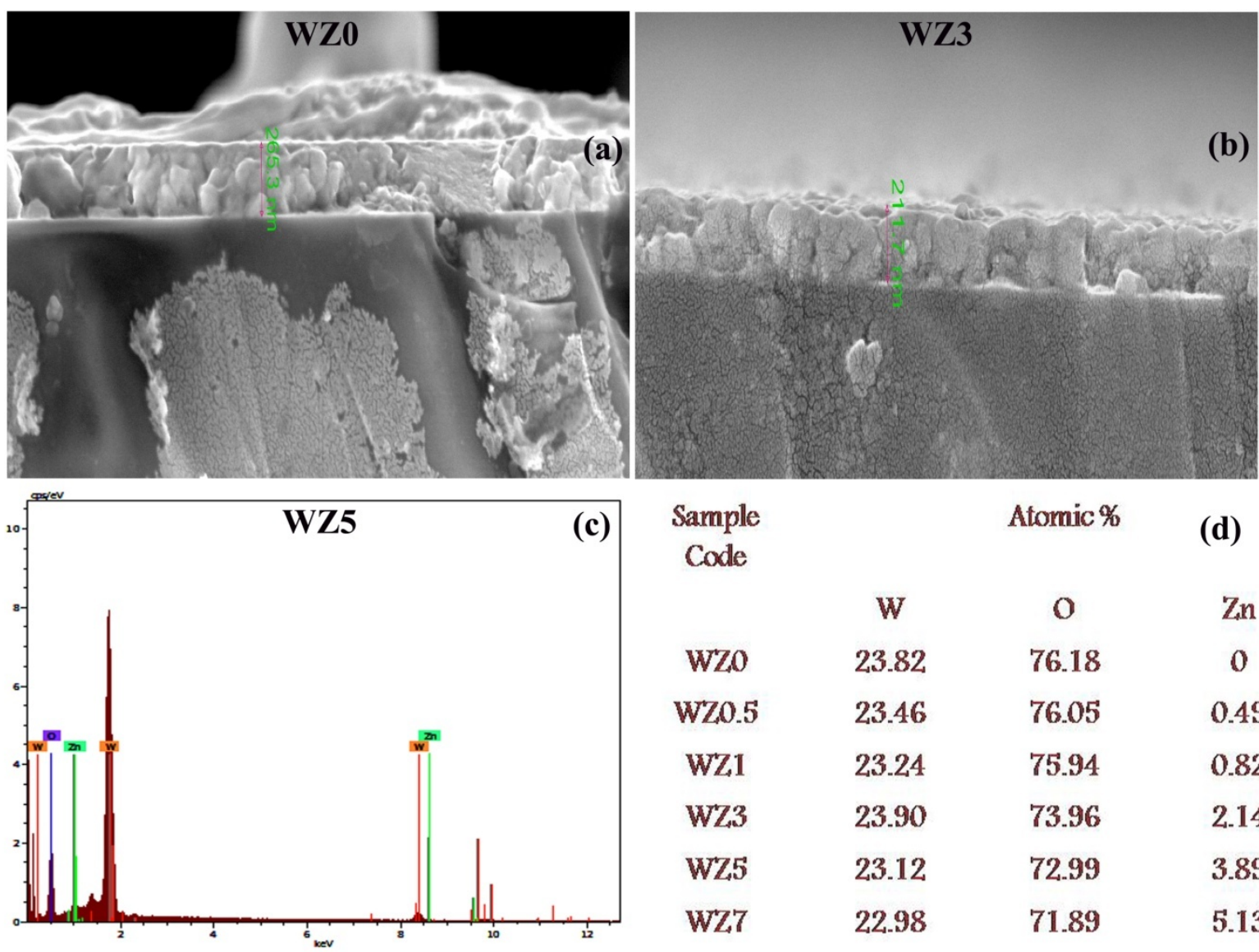

Atomic\%

(d)

$$
\text { Code }
$$

Fig. 7. (a) and (b):Typical lateral FESEM images of WZ0 and WZ3 films respectively; (c): EDX spectrum of WZ5 film; (d):Table showing the atomic percentage of constituents elements in the pure and $\mathrm{ZnO}$ doped $\mathrm{WO}_{3}$ films

The surface morphology of the pure and ZnO doped films was analysed using FESEM micrographs. Fig .6 shows the FESEM micrographs of the pure and $\mathrm{ZnO}$ doped $\mathrm{WO}_{3}$ thin films. The FESEM image of the pure film shows a smooth surface morphology consisting of smaller grains whereas the SEM images of doped films present a compact distribution of well-defined grains with different grain sizes. This non-uniform grain growth in the prepared film may be due to the non-uniform distribution of sputtered molecules and clusters on the growing surface [49]. Increase in $\mathrm{ZnO}$ doping concentration enhances grain growth in a direction normal to the film surface. Among the deposited films WZ5 film presents larger grains with clear grain boundaries. This observation also supports our earlier observation from the XRD analysis that WZ5 film shows the highest crystalline quality. Lateral FESEM images are taken to calculate the thickness of the films. Fig. 7(a) and (b) show lateral FESEM images of two typical films WZ0 and WZ3. Pure film shows highest value for film thickness ( $265 \mathrm{~nm}$ ) compared to $\mathrm{ZnO}$ incorporated films (Table 1). To study the elemental composition of the prepared films, typical energy dispersive X-ray (EDX) spectra is taken. Fig. 7(c) shows a typical EDX spectrum of $5 \mathrm{wt} \% \mathrm{ZnO}$ doped $\mathrm{WO}_{3}$ film. Fig. 7(d) presents the atomic percentage of elements in the prepared films detected using EDX spectra. The EDX spectra of the pure film (Fig. 7(d)) shows the peaks of W and O only. The observation of $\mathrm{Zn}$ peaks in the $\mathrm{ZnO}$ doped films confirms the incorporation of $\mathrm{Zn}$ in the prepared films. In the spectra of all the films the peak due to $\mathrm{W} M \alpha_{1}$ is observed with large intensity. This can be due to the 
superposition of the emitted X-rays of $\mathrm{Si} K \alpha_{1,2}$ lines from the quartz substrate with $\mathrm{W} M \alpha_{1}$ line from the deposited film [50]. Also from Fig. 7(d) we can see that with increase in ZnO doping concentration the atomic percentage of oxygen decreases which is in agreement with the earlier observation from XRD, micro-Raman and XPS analysis

\section{Conclusion}

Highly ordered $\mathrm{ZnO}$ doped $\mathrm{WO}_{3}$ thin films with superior crystalline quality suitable for optoelectronic applications are prepared using RF magnetron sputtering technique. An enhancement in crystalline quality is observed with increase in $\mathrm{ZnO}$ doping concentration up to $5 \mathrm{wt} \%$, beyond which a reduction in crystallinity is observed with increase in $\mathrm{ZnO}$ doping concentration. XRD and Raman analysis reveal the presence of a monoclinic $\mathrm{WO}_{3}$ phase in pure and $\mathrm{ZnO}$ lightly doped $\mathrm{WO}_{3}$ films. The $\mathrm{WO}_{3}$ films with higher $\mathrm{ZnO}$ doping concentrations show the coexistence of monoclinic $\mathrm{WO}_{3}$ and monoclinic $\mathrm{W}_{18} \mathrm{O}_{49}$ phases. The shift in the position of the most intense diffraction peak towards lower diffraction angle suggests the substitutional incorporation of $\mathrm{Zn}$ in $\mathrm{WO}_{3}$ lattice. The observed increase in the values of lattice parameters suggest the expansion of lattice due to the substitutional incorporation of $\mathrm{Zn}$ ions of higher ionic radii in the sites of $\mathrm{W}^{6+}$ ions of lower ionic radii. The crystallite size determination using Scherrer equation and W-H plots point to the presence of considerable lattice strain in the deposited films. XPS spectra also confirm the coexistence of monoclinic $\mathrm{WO}_{3}$ and monoclinic $\mathrm{W}_{18} \mathrm{O}_{49}$ phases in heavily $\mathrm{ZnO}$ doped films. The surface morphology analysis using FESEM reveals a smooth surface morphology with dense distribution of smaller grains in the pure $\mathrm{WO}_{3}$ films whereas the $\mathrm{ZnO}$ doped films presents a surface morphology with dense distribution of bigger grains. EDX spectra also support the incorporation of $\mathrm{ZnO}$ in the doped films.

\section{Conflict of interest}

“The authors declares that we have no conflict of interest”

\section{References}

[1]. R. Sivakumar, A. Moses Ezhil Raj, B. Subramanian, M. Jayachandran, D.C. Trivedi and C. Sanjeeviraja, Mater. Res. Bull., 39, 1479 (2004) .

[2]. C. V. Ramana, S. Utsunomiya, R. C. Ewing, C. M. Julien and U. Becker, J. Phys. Chem. B, 110, 10430(2006).

[3]. T. Pauporte', M. C. Bernard, Y. Soldo-Olivier, and R. Faure, J. Electrochem. Soc., 151 (1), H21(2004).

[4]. M. Sasani Ghamsari, S. Radiman, M. Azmi Abdul Hamid, S. Mahshid and Sh. Rahmani, Mater. Lett., 92, 287 (2013).

[5]. Y.M. Hunge, M.A. Mahadik, S.S. Kumbhar, V.S. Mohite, K.Y. Rajpure, N.G. Deshpande, A.V. Moholkar and C.H. Bhosale, Ceram. Int., 42, 789(2016).

[6]. R. Jolly Bose, Navas Illyasukutty, K.S. Tan, R.S. Rawat, Murukesan Vadakke Matham, Heinz Kohler and V.P. Mahadevan Pillai, Appl. Surf. Sci., 440, 320 (2018).

[7]. A. Rougier, F. Portemer, A. Quede and M. El Marssi, Appl. Surf. Sci., 153, 1(1999). 
[8]. Ahmad Al-Mohammad, phys. stat. sol. (a) 205, 2880 (2008).

[9]. P. TaÈgtstroÈm and U. Jansson, Thin Solid Films, 352, 107 (1999).

[10]. R. Jolly Bose, V.S. Kavitha, C. Sudarsanakumar and V.P. Mahadevan Pillai, Appl. Surf. Sci., 379, 505 (2016).

[11]. Jinzhan Su, Xinjian Feng, Jennifer D. Sloppy, Liejin Guo, and Craig A. Grimes, Nano Lett., 11, 203(2011).

[12]. S. Poongodi, Palaniswamy Suresh Kumar, D. Mangalaraj, N. Ponpandian, P. Meena, Yoshitake Masuda and Chongmu Lee, J. Alloys Compd., 719, 71 (2017).

[13]. Simona Badilescu and P.V. Ashrit, Solid State Ionics, 158, 187 (2003).

[14]. Zhihui Jiao, Jinmin Wang, Lin Ke, Xiao Wei Sun, and Hilmi Volkan Demir, ACS Appl. Mater. Interfaces, 3, 229(2011).

[15]. Rui Liu, Yongjing Lin, Lien-Yang Chou, Stafford W. Sheehan, Wangshu He, Fan Zhang, Harvey J. M. Hou, and Dunwei Wang, Angew. Chem. Int. Ed., 50, 499 (2011)

[16]. K. J. Lethy, D. Beena, V. P. Mahadevan Pillai and K. A. Suresh, J. Nanosci. Nanotechnol., 9, 5335 (2009).

[17]. K.J. Lethy, D. Beena, R. Vinod kumar, V.P. Mahadevan pillai, V. Ganesan,V. Sathe, and D.M. Phase, Appl. Phys. A 91, 637 (2008).

[18]. R. Jolly Bose, R. Vinod Kumar, S. K. Sudheer,V. R. Reddy,V. Ganesan, and V. P. Mahadevan Pillai, J. Appl. Phys., 112, 114311 (2012).

[19]. Gang Li, Xuebin Zhu, Xianwu Tang, Wenhai Song, Zhaorong Yang, Jianming Dai, Yuping Sun, Xu Pan and Songyuan Dai, J. Alloys Compd., 509, 4816 (2011).

[20]. A. Van der Drift, Philips Res. Rep., 22, 267 (1967).

[21]. Mustafa Öztas and Metin Bedir, Thin Solid Films, 516, 1703 (2008).

[22]. L. El Mir, Z. Ben Ayadi, M. Saadoun, K. Djessas, H.J. von Bardeleben and S. Alaya, Appl. Surf. Sci., 254, 570 (2007).

[23]. B. D. Cullity, Elements of X-ray Diffraction (Addison-Wesley Publishing Co., Inc., Massachusetts, 1956), p. 99.

[24]. R.W. Kelsall, I.W Hamley and M. Geoghegan, Nanoscale Science and Technology (New York: Wiley, 2006), p. 265

[25]. S.K. Neogi, S Chattopadhyay, Aritra Banerjee, S.Bandyopadhyay, A. Sarkar and Ravi Kumar, J. Phys.: Condens. Matter., 23, 205801 (2011).

[26]. P. Bindu and Sabu Thomas, J Theor Appl Phys 8, 123 (2014).

[27]. G.K. Williamson and W. H. Hall, ActaMetall.,1, 22, (1953).

[28]. S. B. Qadri, J. P. Yang, E. F. Skelton, and B. R. Ratna, Appl. Phys. Lett., 70, 1020 (1997).

[29]. X.F. Cheng, W.H. Leng, D.P. Liu, J.Q. Zhang and C.N. Cao, Chemosphere, 68, 1976 (2007).

[30]. A. R. Denton, N. W. Ashcroft, Phys. Rev. A 43, 3161(1991).

[31]. Bharati Panigrahy, M.Aslam, and D. Bahadur, J. Phys. Chem. C, 114, 11758(2010). 
[32]. Navas Illyaskutty, Sreeja Sreedhar, Heinz Kohler,Reji Philip, Vinodkumar Rajan and V. P. Mahadevan Pillai, J. Phys. Chem. C, 117, 7818 (2013).

[33]. Gao Li and Zhang Jian-Min, Chinese Phys. B, 18, 4536 (2009).

[34]. E. SALJE, Acta Cryst. A 31, 360(1975).

[35]. V. Ya kabanov and V. F. Chuvaev, Russ. J. Phys. Chem. 38,717 (1964)

[36]. I. R. Beattie and T. R. Gilson, J. Chem. Soc.(A), 2322 (1969).

[37]. Joel Díaz-Reyes, Roberto Castillo-Ojeda, Miguel Galván-Arellano and Orlando Zaca-Moran1, Adv. Condens. Matter Phys.,2013,1(2013).

[38]. O. Pyper, A. Kaschner and C. Thomsen, Sol. Energy Mater. Sol. Cells 71, 511(2002).

[39]. M. Breedon, P. Spizzirri, M. Taylor, J. Plessis, D. McCulloch, J. Zhu, L. Yu, Z.Hu, C. Rix, W. Wlodarski and K. Kalantar-Zadeh, Cryst. Growth Des., 10, 430 (2010).

[40]. G. Fang, Z. Liu, K. L. Yao, J. Phys. D: Appl. Phys., 34, 2260 (2001).

[41]. N. Naseri, R. Azimirad, O. Akhavan and A.Z. Moshfegh, Thin Solid Films, 518, 2250 (2010).

[42]. M. Feng, A. L. Pan, H. R. Zhang, Z. A. Li, F. Liu, H. W. Liu, D. X. Shi,B. S. Zou, and H. J. Gao, Strong photoluminescence of nanostructured crystalline tungsten oxide thin films, Appl. Phys. Lett. 86, 141901 (2005).

[43]. Wen-Jing Li, Zheng-Wen Fu, Appl. Surf. Sci., 256, 2447 (2010).

[44]. Chongshen Guo, Shu Yin, Yunfang Huang, Qiang Dong and Tsugio Sato, Langmuir, 27, 12172 (2011).

[45]. B.A. De Angelis and M. Schiavello, J. Solid State Chem., 21, 67 (1977).

[46]. Yanlong Yu, Jingsheng Wang, Wei Li, Wenjun Zheng and Yaan Cao, CrystEngComm,17,5074 (2015).

[47]. Seung-Ki Jeong, Myeong-Ho Kim, Sang-Yeon Lee, Hyungtak Seo and Duck-Kyun Choi, Nanoscale Res. Lett., 9, 1 (2014).

[48]. Nur Hidayatul Nazirah Kamarudin, Aishah Abdul Jalil, Sugeng Triwahyono,Rino R. Muktic, Muhammad Arif Ab Aziz, Herma Dina Setiabudi, Mohd Nazlan Mohd Muhid and Halimaton Hamdan, Appl. Catal., A, 431-432, 104 (2012).

[49]. S.R. Chalana, R.Vinodkumar, I.Navas, V.Ganesan and V.P. MahadevanPillai, J.Lumin., 132, 944 (2012).

[50]. J.A. Bearden and A.F. Burr, Rev. Mod. Phys., 39 125(1967). 